\title{
THE
}

$12-1988$

\section{High-Temperature Spin Dynamics of the Classical Heisenberg Magnet in One, Two, Three and Infinite Dimensions}

Gerhard Müller

University of Rhode Island, gmuller@uri.edu

Follow this and additional works at: https://digitalcommons.uri.edu/phys_facpubs

Terms of Use

All rights reserved under copyright.

\section{Citation/Publisher Attribution}

Gerhard Müller. High-temperature spin dynamics of the classical Heisenberg magnet in one, two, three and infinite dimensions. J. Physique C8 (1988), 1403-1404.

Available at: http://dx.doi.org/10.1051/jphyscol:19888644

This Article is brought to you for free and open access by the Physics at DigitalCommons@URI. It has been accepted for inclusion in Physics Faculty Publications by an authorized administrator of DigitalCommons@URI. For more information, please contact digitalcommons-group@uri.edu. 


\title{
High-Temperature Spin Dynamics of the Classical Heisenberg Magnet in One, Two, Three and Infinite Dimensions
}

\author{
Gerhard Müller \\ Department of Physics, University of Rhode Island, Kingston RI 02881, USA
}

\begin{abstract}
Time-dependent spin-autocorrelation functions at $T=\infty$ for the classical Heisenberg magnet in dimensions $d=1,2$ and 3 are investigated by computer simulations. For the equivalent-neighbor Heisenberg model, the autocorrelation function is calculated analytically.
\end{abstract}

Local spin fluctuations in magnetic insulators at high temperature spread rather slowly and persist over appreciable times and distances only if a conservation law prohibits them from decaying [1]. The transport of spin fluctuations is then governed by a diffusive process, which is directly observable by dynamical experiments on insulating magnetic compounds [2]. This paper reports an approach to computer simulations for the determination of time-dependent correlation functions of classical spin systems which differs from methods previously employed $[2,3]$. It is tailored to situations where the coherence length is small or, in fact, zero. The focus here is on spinautocorrelation functions $C_{0}(t) \equiv\left\langle\mathbf{S}_{j}(t) \cdot \mathbf{S}_{j}(0)\right\rangle$ at $T=\infty$ for the classical Heisenberg model

$$
H=-J \sum_{\langle i, j\rangle} \mathbf{S}_{i} \cdot \mathbf{S}_{j}
$$

with nearest-neighbor coupling. The variables $\mathbf{S}_{j}$ are classical 3-component unit vectors on a $d$ dimensional hypercubic lattice with periodic boundary conditions. The classical spin dynamics is specified by the equations of motion

$$
\dot{\mathbf{S}}_{j}=-\mathbf{S}_{j} \times \partial H / \partial \mathbf{S}_{j}
$$

For the numerical determination of the infinite temperature autocorrelation function, choose the $k$-th set of initial conditions $\left\{\mathbf{S}_{i}^{(k)}\right\}$ at random and integrate the equations of motion (2) numerically over some time interval [4]. $C_{0}(t)$ is then evaluated as an average of $\mathbf{S}_{j}(t) \cdot \mathbf{S}_{j}^{(k)}$ over all $N$ lattice sites and over $K$ randomly chosen initial spin configurations. Results which are meaningful for the infinite system can then be obtained by choosing both $N$ and $K$ sufficiently large. In view of the fact that the transport of spin fluctuations is slow at high temperatures, the most efficient and accurate method is first to reduce the statistical fluctuations by extensive ensemble averaging and then to eliminate finite-size effects by analyzing precise data systematically for increasing $N$. Results of such a simulation are displayed in figure 1 . The three curves which are decorated by full symbols represent the autocorrelation functions $C_{0}(t)$ at $T=\infty$ of the $1 \mathrm{~d}, 2 \mathrm{~d}$ and $3 \mathrm{~d}$ Heisenberg models on lattices of size $N=50$ (1d), $N=20 \times 20$ (2d, square lattice) and $N=8 \times 8 \times 8$ (3d, simple cubic lattice), respectively. The time axis is rescaled by $\sqrt{d}$, which causes the three curves to superimpose very precisely for $J t / \sqrt{d} \leq 1.7$. In all three dimensionalities, $C_{0}(t)$ is characterized by a diffusive long-time tail. Its strength weakens with increasing $d$ in qualitative agreement with the phenomenological spin diffusion result $C_{0}^{(\mathrm{SD})}(t) \sim t^{-d / 2}$. A more detailed analysis of these diffusive tails has revealed that the long-time tails of the simulation data are indeed of the power-law type, $t^{-\alpha_{d}}$, but with exponents $\alpha_{d}$ which are in excess of $d / 2: \alpha_{1}=-0.609 \pm 0.005, \alpha_{2}=1.050 \pm 0.025$, $\alpha_{3} \simeq 1$.6. This manifestation of anomalous spin diffusion was emphasized in reference [5]. 


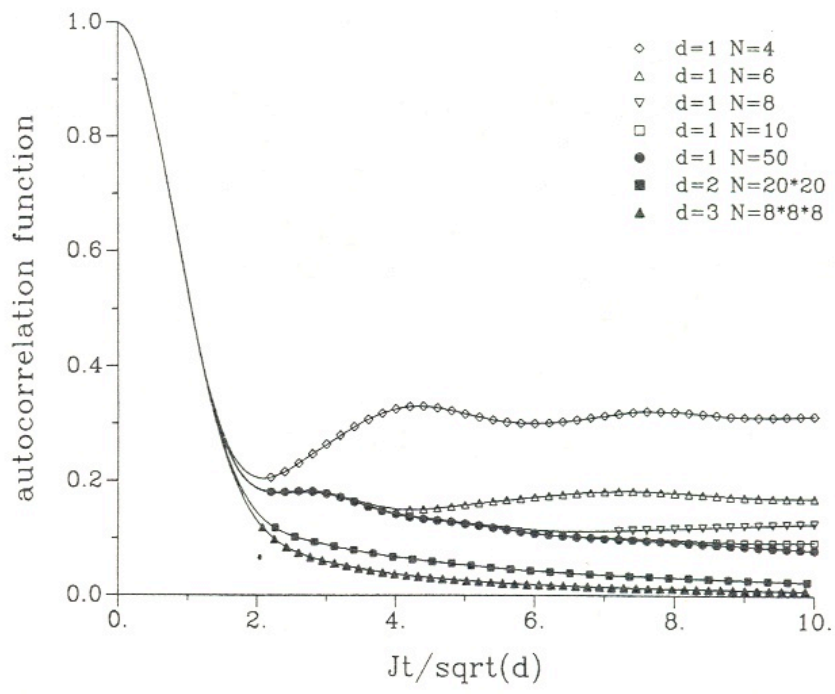

Figure 1. Spin-autocorrelation function $C_{0}(t)=\left\langle\mathbf{S}_{j}(t) \cdot \mathbf{S}_{j}(0)\right\rangle$ at $T=\infty$ of the classical Heisenberg model with nearest-neighbor interaction. The curves with open symbols represent simulation data averaged over $K=20000$ random initial conditions for 1 d systems of sizes $N=4,6,8,10$. The curves with full symbols show results for a linear chain $(N=50)$, a square lattice $(N=20 \times 20)$, and a simple cubic lattice $(N=8 \times 8 \times 8)$. They represent simulation data averaged over $K=22792$ (1d), $K=4563$ (2d), and $K=4041$ (3d) random initial conditions.

The curves in figure 1 which are decorated by open symbols represent the autocorrelation functions of chains with $N=4,6,8$ and 10 spins. For small $t$ all four curves lie on top of each other and on top of the curve representing a chain of length $N=50$. The curve for $N=4$ is the first to deviate from the others (at $J t \simeq 1$ ) followed by the curve representing the next longer chain $(N=6)$ somewhat later $(J t \simeq 3)$ etc. For all finite $N$, the function $C_{0}(t)$ decays to a positive constant as $t \rightarrow \infty$; that constant is a monotonically decreasing function of $N$ which tends to zero as $N \rightarrow \infty$. For $N=50$ no noticeable finite-size effects occur out to $J t \simeq 40$, i.e. much beyond the maximum time lag shown in figure 1 . Likewise, the $2 \mathrm{~d}$ and $3 \mathrm{~d}$ autocorrelation functions to not exhibit any significant finite-size effects on the time intervals for which data are shown in figure 1.

The autocorrelation function of the equivalent-neighbor Heisenberg model (Kittel-Shore model) can be determined exactly. The model is specified by Hamiltonian (1) with the sum $\langle i j\rangle$ now taken over all distinct pairs of lattice sites. The equation of motion (2) then has the form $\dot{\mathbf{S}}_{j}=J \mathbf{S}_{j} \times \mathbf{S}_{T}$, where $\mathbf{S}_{T}=\sum_{j} \mathbf{S}_{j}$ is a constant of the motion. The time evolution consists of a simple precession of each spin $\mathbf{S}_{j}$ about the direction of $\mathbf{S}_{T}$, all with the same frequency $J\left|\mathbf{S}_{T}\right|$ [6]. The $T=\infty$ autocorrelation function is then determined by an ensemble average over periodic phase-space trajectories. For $N=2$, the result is [7]

$$
C_{0}(t)=\frac{1}{2}+\frac{3}{2(J t)^{3}} \sin (2 J t)-\frac{3+2(J t)^{2}}{4(J t)^{4}}+\frac{3-4(J t)^{2}}{4(J t)^{4}} \cos (2 J t) .
$$

It is plotted in figure 2 along with the autocorrelation functions for chains with $N=4,6,8$ sites. Here the ensemble averaging was done numerically. We observe that for increasing $N$, the function $C_{0}(t)$ goes through an increasingly deep minimum and then approaches a constant for $t \rightarrow \infty$ which is a monotonically decreasing function of $N$; that constant is $1 / 2$ for $N=2$ and approaches the value $1 / 3$ as $N \rightarrow \infty$. This is in contrast to the sequence of curves for the $1 \mathrm{~d}$ Heisenberg model depicted in figure 1, where the long-time asymptotic value of $C_{0}(t)$ goes to zero as $N \rightarrow \infty$. The different behavior is attributable to the fact that the special linear time evolution of the equivalentneighbor Heisenberg model does not accommodate any process by which local fluctuations spread 
over the entire system. Note that the time axis in figure 2 is rescaled by $\sqrt{N / 2}$, which corresponds to a rescaling of the interaction strength in the equivalent-neighbor model by the same amount $\left(J^{\prime}=J / \sqrt{N / 2}\right)$. The connection to the time scale used in figure 1 is thus established if we note that the coordination number in a $d$-dimensional hypercubic lattice is $2 d$.

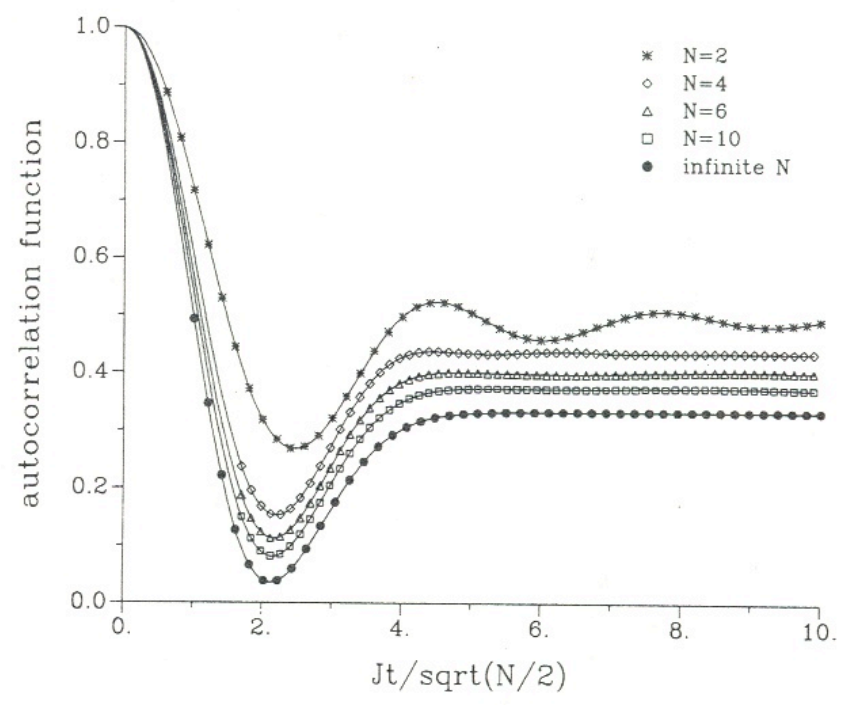

Figure 2. Spin-autocorrelation function $C_{0}(t)=\left\langle\mathbf{S}_{j}(t) \cdot \mathbf{S}_{j}(0)\right\rangle$ at $T=\infty$ of the classical equivalent-neighbor Heisenberg model. The curves for $N=2$ and $N=\infty$ represent exact analytic results. The remaining curves represent ensemble averages over $K=250000(N=4)$, $K=216140(N=6)$, and $K=133151(N=10)$ random initial conditions.

For all finite $N$, the autocorrelation function of the equvalent-neighbor model approaches its long-time asymptotic value in a power-law type way: $\sim \cos \left(\omega_{N} t\right) t^{-\alpha_{N}}$. For increasing $N$, the characteristic exponent $\alpha_{N}$ becomes larger and larger, reflecting the fact that the (power-law) singularities in the intensity spectrum become weaker and weaker as $N \rightarrow \infty$. In the thermodynamic limit, they are wiped out completely, a result dictated by the central-limit theorem. The exact expression for $N \rightarrow \infty$ reads

$$
C_{0}(t)=\frac{1}{3}+\frac{2}{3}\left[1-\frac{2}{3}\left(J^{\prime} t\right)^{2}\right] \mathrm{e}^{-\left(J^{\prime} t\right)^{2} / 3} .
$$

Now $C_{0}(t)$ approaches its long-time asymptotic value in the form of a Gaussian, much more rapidly than for finite $N$. Expression (4) is also shown in figure 2.

Acknowledgment: This work was supported by the U.S. National Science Foundation Grant No. DMR-86-03036. We have used a modified cmpj.sty style file.

\section{References}

1. Forster, D., Hydrodynamic Fluctuations, Broken Symmetry, and Correlation Functions (Benjamin, Reading) 1975.

2. Steiner, M., Villain, J., Windsor, C. G., Adv. Phys. 25 (1976) 87.

3. Lurie, N. A., Huber, D. L., Blume, M., Phys. Rev. B 9 (1974) 2171; Landau, D. P., Thomchik, J., J. Appl. Phys. 50 (1979) 1822.

4. A 4th-order Runge-Kutta integration with fixed time step $J d t=0.005-0.025$ is used.

5. Müller, G., Phys. Rev. Lett. 60 (1988) 2785.

6. Magyari, E., Thomas, H., Weber, R., Kaufman, C., Müller, G., Z. Phys. B 65 (1987) 363.

7. Srivastava, N., Kaufman, C., Müller, G., J. Appl. Phys. 63 (1988) 4154. 